\title{
Effect of vedolizumab (anti- $\alpha 4 \beta 7$-integrin) therapy on histological healing and mucosal gene expression in patients with UC
}

\author{
Ingrid Arijs, ${ }^{1,2,3}$ Gert De Hertogh, ${ }^{4}$ Bart Lemmens, ${ }^{4}$ Leentje Van Lommel, ${ }^{5}$ \\ Magali de Bruyn, ${ }^{1,6}$ Wiebe Vanhove, ${ }^{1}$ Isabelle Cleynen, ${ }^{1,7}$ Kathleen Machiels, ${ }^{1}$ \\ Marc Ferrante, ${ }^{1,7}$ Frans Schuit, ${ }^{5}$ Gert Van Assche, ${ }^{1,8}$ Paul Rutgeerts, ${ }^{1,8}$ \\ Severine Vermeire ${ }^{1,8}$
}

- Additional material is published online only. To view please visit the journal online (http://dx.doi.org/10.1136/ gutjnl-2016-312293)

For numbered affiliations see end of article.

Correspondence to Dr Severine Vermeire, TARGID, KU Leuven, Herestraat 49, O\&N1, mailbox 701, Leuven B-3000, Belgium; severine.vermeire@uzleuven.be

Received 20 May 2016 Revised 12 September 2016 Accepted 20 September 2016 Published Online First 7 October 2016
CrossMark

To cite: Arijs I, De Hertogh $G$ Lemmens B, et al. Gut 2017:67:43-52.

\section{ABSTRACT}

Objective Lymphocyte recruitment to the inflamed gut is increased in UC. Inhibition of this cell trafficking by vedolizumab (VDZ) was successful in inducing and maintaining remission and in induction of endoscopic mucosal healing. There are no data on histological healing with VDZ. We studied histological changes following VDZ therapy and compared gene expression in patients with UC before and after therapy.

Design Forty-one patients with UC from GEMINI I and LTS were studied before and at three time points (weeks 6/12/52) following VDZ therapy. Colonic biopsies were scored using the Geboes index and correlated with Mayo endoscopic subscore. Gene expression was analysed using Affymetrix gene arrays.

Results Fifty-five per cent of patients achieving endoscopic healing (= Mayo endoscopic subscore $0-1$ ) with VDZ at the studied time points also had histological healing (= Geboes grade $0-1)$. In most healers, some residual histological changes (eg, disturbed architecture and increased mononuclear cell infiltrate) were still observed, although this was less at week 52. VDZ restored expression of many inflammatory genes in patients with endoscopic healing only at week 52 and not before. In VDZ healers, the expression of many genes remained dysregulated at weeks 6/12/52 compared with controls.

Conclusions VDZ induces histological healing in $>50 \%$ of patients with endoscopic healing, with maximal effect at week 52. VDZ also restored, although incompletely, the colonic expression of many immunerelated genes in patients with UC achieving endoscopic healing at week 52. However, persistent histological and gene dysregulations did remain even in healers, suggesting that maintenance therapy will be necessary to control the intestinal inflammation.

Trial registration numbers: NCT00783718 and NCT00790933; post-results.

\section{INTRODUCTION}

IBDs are characterised by a continuous influx of leucocytes from the blood circulation into the inflamed gut. This migration of $\mathrm{CD}^{+} \mathrm{T}$ lymphocytes is strictly regulated by cell adhesion molecules and a sequential upregulation of selectins and later integrins that will interact and bind to their respective receptor mucosal vascular addressin cell

\section{Significance of this study}

What is already known on this subject?

- Vedolizumab (anti- $\alpha 4 \beta 7$-integrin) is the first antiadhesion therapy in UC and is efficacious in inducing and maintaining clinical remission, and in induction of endoscopic healing.

- Although endoscopic mucosal healing is now considered as the treatment goal in IBD, it is not curative and relapses are still observed in a significant proportion of patients, even when the treatment is continued.

- Clinicians currently explore whether histological healing may be a better therapeutic end point.

- The importance of histological healing has previously been demonstrated for anti-tumour necrosis factor therapy (eg, infliximab, adalimumab and golimumab) in UC.

\section{What are the new findings?}

- A significant proportion of patients with UC who achieve endoscopic healing with vedolizumab also had histological healing, with a maximal effect seen at week 52.

- Vedolizumab restored, although incompletely, the colonic expression of many immune-related genes in patients with UC achieving endoscopic healing, and this was only observed at week 52 and not before.

- Persistent histological and immune-related gene expression abnormalities remain even in patients achieving mucosal healing with vedolizumab and give insight into the underlying pathogenic mechanisms.

How might it impact on clinical practice in the foreseeable future?

- This study shows for the first time histological improvement following vedolizumab therapy. A maximal effect of vedolizumab on histology and mucosal gene expression was seen at week 52 , and these findings help us to understand the relative slow onset of clinical efficacy of vedolizumab.

- The identified persistence of abnormalities in patients with UC, despite achievement of mucosal healing, might explain why mucosal lesions rapidly recur if patients do not receive maintenance therapy. This also suggests that maintenance therapy will be necessary to control intestinal inflammation. 
adhesion molecule 1 (MAdCAM-1) on the vascular endothelium. Vedolizumab (VDZ; Entyvio; Takeda Pharmaceuticals, Deerfield, Illinois, USA) is a humanised monoclonal antibody targeting $\alpha 4 \beta 7$-integrin, which is almost uniquely expressed on gut-homing lymphocytes, and thereby selectively blocks lymphocyte trafficking to the gut. Following successful completion of the required randomised-controlled GEMINI studies, VDZ got approval for Crohn's disease (CD) and UC by the US Food and Drug Administration and the European Medicines Agency. Both GEMINI I (UC) and II (CD) showed that VDZ is efficacious in inducing and maintaining clinical remission. ${ }^{1} 2$ GEMINI I also demonstrated significant mucosal healing in patients with UC.

Although mucosal healing (often defined as disappearance of all ulcerations during endoscopy) is considered nowadays as the treatment goal in IBD ${ }^{3}$ it is not certain whether this is sufficient as an end point. Other therapies associated with significant healing, such as infliximab (IFX) and adalimumab, are associated with disease relapse in $50 \%$ of patients when the treatment is discontinued. The triggers for recurrence of inflammation are greatly unknown, although some histopathological features such as persistent basal plasmocytosis have been suggested. ${ }^{4}$ Until now, no data are available with regard to histological changes following VDZ treatment in UC. It is therefore unknown whether endoscopic healing observed after successful VDZ therapy also correlates with a reduction of the inflammatory infiltrate at the histological level and in what timeframe these changes occur.

To obtain more insights into this question, we studied histological changes before and after start of VDZ and correlated these findings with the Mayo endoscopic subscore. Furthermore, we investigated the effect of VDZ on the colonic mucosal gene expression in these patients and compared results with what we observed with IFX therapy in UC.

\section{METHODS}

\section{Patients and biopsy specimens}

The study was carried out at the University Hospitals Leuven (Leuven, Belgium). The characteristics of patients and controls are summarised in table 1. Endoscopic-derived biopsies were collected from patients with UC during two phase III trials of VDZ, GEMINI I and GEMINI LTS. ${ }^{1}$ Gemini I was a phase III randomised, placebo-controlled, double-blinded, multicentre study investigating induction and maintenance of clinical response and remission by VDZ in patients with moderate-to-severe UC, and Gemini LTS is an ongoing multicentre, open-label study on long-term safety and efficacy of VDZ in patients with UC and CD. Altogether 44 patients who participated in these studies at our centre (31 patients from Gemini I and 13 from Gemini LTS) were included, and 41 out of 44 patients were treated with VDZ at inclusion. Biopsies were taken at protocol-specified time points (week (W) 0, W6, W12 and W52, or at study withdrawal). A total of 120 colonic mucosal biopsies were available for analysis.

As control groups, colonic mucosal biopsies were collected from 23 patients with UC before and W4-6 after first IFX therapy as well as from 12 non-IBD control individuals with normal mucosa.

Biopsies were taken in the colon at the edge of ulcers whenever present. If no ulcers were seen, then biopsies were taken at the most inflamed colon segment. Half of the biopsies were immediately snap-frozen in liquid nitrogen and stored at $-80^{\circ} \mathrm{C}$ for gene expression analysis. The remaining biopsies were fixed in Carnoy's or formalin's fixative for up to 5 hours and then
Table 1 Baseline characteristics of the study population

\begin{tabular}{|c|c|c|c|}
\hline $\begin{array}{l}\text { Baseline } \\
\text { characteristics }\end{array}$ & UC VDZ (n=41) & UC IFX (n=23) & Controls $(n=12)$ \\
\hline Male/female (\%) & $21 / 20(51.2 / 48.8)$ & $13 / 10(56.5 / 43.5)$ & $6 / 6(50 / 50)$ \\
\hline $\begin{array}{l}\text { Median (IQR) age } \\
\text { (years) }\end{array}$ & $40.5(32-49.4)$ & $41.3(31.1-49.6)$ & $68.2(59-72.7)$ \\
\hline $\begin{array}{l}\text { Median (IQR) duration } \\
\text { of disease (years) }\end{array}$ & $10.2(4.4-14.6)$ & $7.6(2.8-17.2)$ & NA \\
\hline \multicolumn{4}{|l|}{ Extent of disease } \\
\hline $\begin{array}{l}\text { UC left-sided colitis/ } \\
\text { pancolitis (\%) }\end{array}$ & $18 / 23(43.9 / 56.1)$ & 6/17 (26.1/73.9) & NA \\
\hline \multicolumn{4}{|l|}{ Histology (Geboes score) } \\
\hline $0-1(\%)$ & $0(0)$ & $0(0)$ & NA \\
\hline $2-5(\%)$ & $41(100)$ & $23(100)$ & NA \\
\hline \multicolumn{4}{|c|}{ Mayo endoscopic subscore } \\
\hline $0-1(\%)$ & $0(0)$ & $0(0)$ & NA \\
\hline $2-3(\%)$ & $41(100)$ & $23(100)$ & NA \\
\hline $\begin{array}{l}\text { Median (IQR) total } \\
\text { Mayo score }\end{array}$ & $10(8-11)$ & $10(9-10)$ & NA \\
\hline \multicolumn{4}{|l|}{ Medication (\%) } \\
\hline 5-Aminosalicylates & $29(70.7)$ & $18(78.3)$ & NA \\
\hline Corticosteroids & $17(41.5)$ & $7(30.4)$ & NA \\
\hline $\begin{array}{l}\text { Azathioprine/ } \\
\text { 6-mercaptopurine }\end{array}$ & $7(17.1)$ & $14(60.8)$ & NA \\
\hline Methotrexate & $1(2)$ & $0(0)$ & NA \\
\hline Anti-TNF & $0(0)$ & $0(0)$ & NA \\
\hline Active smoking (\%) & $5(12.2)$ & $2(8.3)$ & $0(0)$ \\
\hline
\end{tabular}

dehydrated, cleared and paraffin-embedded for histological examination.

\section{Definitions of histological and endoscopic healing}

$\mathrm{H} \& \mathrm{E}$ stained slides from the paraffin blocks of each patient were scored blindly for features of chronic intestinal inflammation using the histological scoring system of Geboes et al. ${ }^{5}$ Histological mucosal healing was defined as a grade 0 or 1 on the Geboes score, ${ }^{5}$ and endoscopic mucosal healing was defined as a Mayo endoscopic subscore of 0 or $1 .^{6}$

\section{Mucosal gene expression analysis}

Total RNA was isolated from the fresh-frozen biopsies using the RNeasy Mini Kit (Qiagen, Benelux BV, Venlo, the Netherlands). As previously described, ${ }^{7}$ total RNA (150 ng) was analysed for whole-genome gene expression analysis via Affymetrix GeneChip Human Gene 1.0 ST arrays (Affymetrix, Santa Clara, California, USA), according to the Affymetrix technical manual 4425209 Rev.B. The microarray data are available at the Gene Expression Omnibus database under accession number GSE73661 (http://www.ncbi.nlm.nih.gov/geo/query/acc.cgi? token $=$ ebcdycqsxfcdrap\&acc $=$ GSE73661).

For the gene expression studies, response to therapy was defined as endoscopic mucosal healing and assessed for VDZ at W6, W12 and W52, and for IFX at W4-6.

The microarray data were analysed in R (http://www.r-project. org/). The Affymetrix raw gene array data (.CEL files) were processed to obtain a $\log 2$ expression value for each gene probe set using the robust multichip average (RMA) method implemented in the aroma.affymetrix R package. ${ }^{8}$ A non-specific filtering was performed on the $\log 2$ RMA normalised data from all the samples, and only gene probe sets with an intensity $>\log 2(50)$ in at least $1 \%$ of the samples and an IQR of $\log 2$ intensities across 
the samples $>0.5$ were included, leaving 5885 (/33252) gene probe sets for further data analysis. Principal component analysis (PCA) was performed on the normalised and filtered $\log 2$ microarray data of control samples, pretreatment and post-treatment UC samples. For comparative analysis, the LIMMA package ${ }^{9}$ was used to identify the filtered gene probe sets that showed significant differential expression between the studied groups, based on moderated t-statistics with Benjamini-Hochberg false discovery rate (FDR) correction for multiple testing. Gene probe sets were selected as biologically significant using FDR $<0.05$ and a fold change $(\mathrm{FC})>2$. The Bio Functional Analysis tool in the Ingenuity Pathway Analysis program (Ingenuity Systems, http:// www.ingenuity.com) was used to identify the biological (sub-) functions associated with the data sets of significantly differentially expressed gene probe sets.

To confirm the microarray data, quantitative RT-PCR was performed for selected genes (LPHN2, FGF7, MADCAM1, INDO and $I L 8$ ), and $\beta$-actin (as endogenous reference gene) on the RNA samples, as previously described. ${ }^{10}$ Primer and probe sequences are summarised in online supplementary table S1. Data were analysed according to the Pfaffl method. ${ }^{11}$ Results were analysed in SPSS using Mann-Whitney U test for unpaired samples and Wilcoxon signed-rank test for paired samples. $p$ Value $\leq 0.05$ was considered significant.

\section{Immunohistochemistry}

For a more detailed histological assessment of the biopsy samples from patients showing mucosal healing, immunostains on $5 \mu \mathrm{m}$-thick sections were performed for different types of $\mathrm{T}$ cells $\left(\mathrm{CD} 3^{+} \mathrm{T}\right.$ lymphocytes, $\mathrm{CD} 4^{+} \mathrm{T}$ helper cells and $\mathrm{CD} 8^{+}$ cytotoxic T cells) and for mast cells (c-kit stain). Furthermore, protein localisation of selected genes, namely ITGB7, LPHN2 and MADCAM1, in the colonic mucosa was performed by immunohistochemistry. The protocol details for immunohistochemistry are described in online supplementary file 1. An IBD experienced pathologist $(\mathrm{GDH})$ evaluated all the stains.

\section{RESULTS}

Correlation between endoscopic and histological healing

Overall, when taking all the studied patient biopsy samples ((120 - 1 with no histological information) VDZ+46 IFX cohort) into account, we observed a significant correlation $(\tau=0.709$ and $p<0.001)$ between endoscopic and histological disease activity (healing/no healing) in $91.5 \%$ of the samples $(151 / 165)$. Dissimilarities were mainly present in a few patients showing clear endoscopic but no histological healing.

In the subgroup of patients with endoscopic healing (32/165, including both VDZ and IFX cohorts), 12 or $37.5 \%$ showed persistent histological disease activity, defined as Geboes grades $2-5$, and were therefore not in histological remission. When we looked only to the biopsy samples from patients treated with VDZ who achieved endoscopic mucosal healing (= responders) at the studied time points $(n=22), 55 \%$ of these samples also showed histological healing $(12 / 22 ; 3 / 6$ at W6, 2/3 at W12, 7/ 12 at W52), and only one patient treated with VDZ who had no endoscopic healing (= non-responder) at W12 showed histological healing $(1 / 35 ; 0 / 21$ at W6, 1/10 at W12, 0/4 at W52) (figure 1).

Next, we studied whether the characteristics of histological healing achieved by VDZ at various time points (figure 2) was different to those achieved by IFX at W4-6. To study this, we focused in the (endoscopic and histological) healed biopsy samples on (1) residual architectural disturbance as encoded in the Geboes grade 0 category $(0.0=$ no abnormality, $0.1=$ mild abnormality,

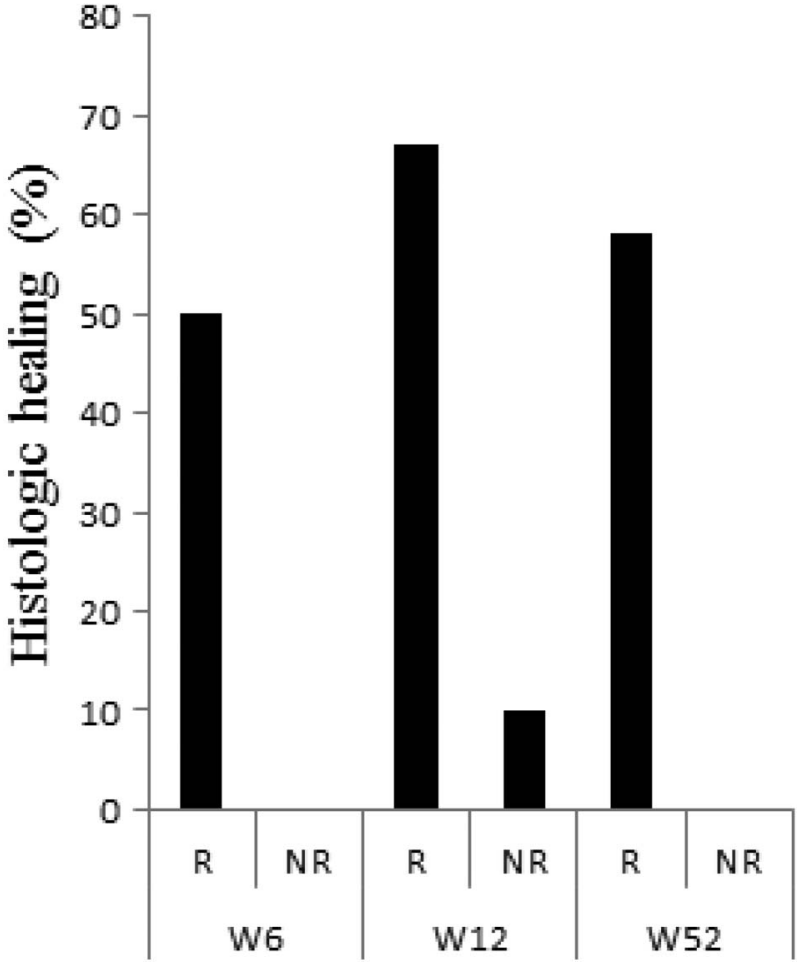

Figure 1 Bar graph representing the percentage of patients showing histological healing in vedolizumab (VDZ) responders ( $R$; patients achieving endoscopic healing after VDZ therapy) and in VDZ non-responders (NR; patients with no endoscopic healing after VDZ therapy) at the studied time points (week (W)6, W12 and W52).

$0.2=$ mild or moderate diffuse or multifocal abnormalities, $0.3=$ severe diffuse or multifocal abnormalities), (2) mononuclear cell infiltrate in the lamina propria (LP) classified by Geboes grade $1(1.0=$ no increase, $1.1=$ mild but unequivocal increase, 1.2=moderate increase, $1.3=$ marked increase), (3) T lymphocytes as identified by CD3 positivity, as these are the major subset of lymphoid cells present in the mononuclear infiltrate, (4) the ratio $\mathrm{CD}^{+} / \mathrm{CD}^{+}$T cells and (5) the LP mast cells (table 2 ).

In patients treated with VDZ who achieved mucosal healing at W6 but also at W12, a variable picture was seen with regard to architectural abnormalities as the grades 0.1 and 0.2 were represented. The same observation was seen for mononuclear cell infiltrates with the grades 1.0 and 1.1. As for the T lymphocytes, a constant influx was observed similar to the findings in the baseline biopsies. By W52, however, these changes had largely disappeared, with generally mild residual architectural abnormalities (mainly grade 0.1 ), no or only a mild increase of the mononuclear cell infiltrate (grade 1.0/1.1) and no or mild increase of the $\mathrm{T}$ lymphocytes. Importantly, in patients who achieved mucosal healing with IFX at W4-6, we also detected a markedly increased mononuclear cell infiltrate (grade 1.1/1.2) and a corresponding increase in $\mathrm{T}$ lymphocytes, with extensive residual architectural abnormalities (grade 0.1/0.2). For all studied time points, no change in the ratio of $\mathrm{CD} 4^{+} / \mathrm{CD} 8^{+} \mathrm{T}$ cells and no effect on mast cell numbers were seen.

\section{Whole-genome gene expression microarray analysis} Distinctive colonic gene expression profiles based on inflammation status

PCA showed a clear separation of the colonic samples based on inflammatory activity (figure 3). The samples from patients with 


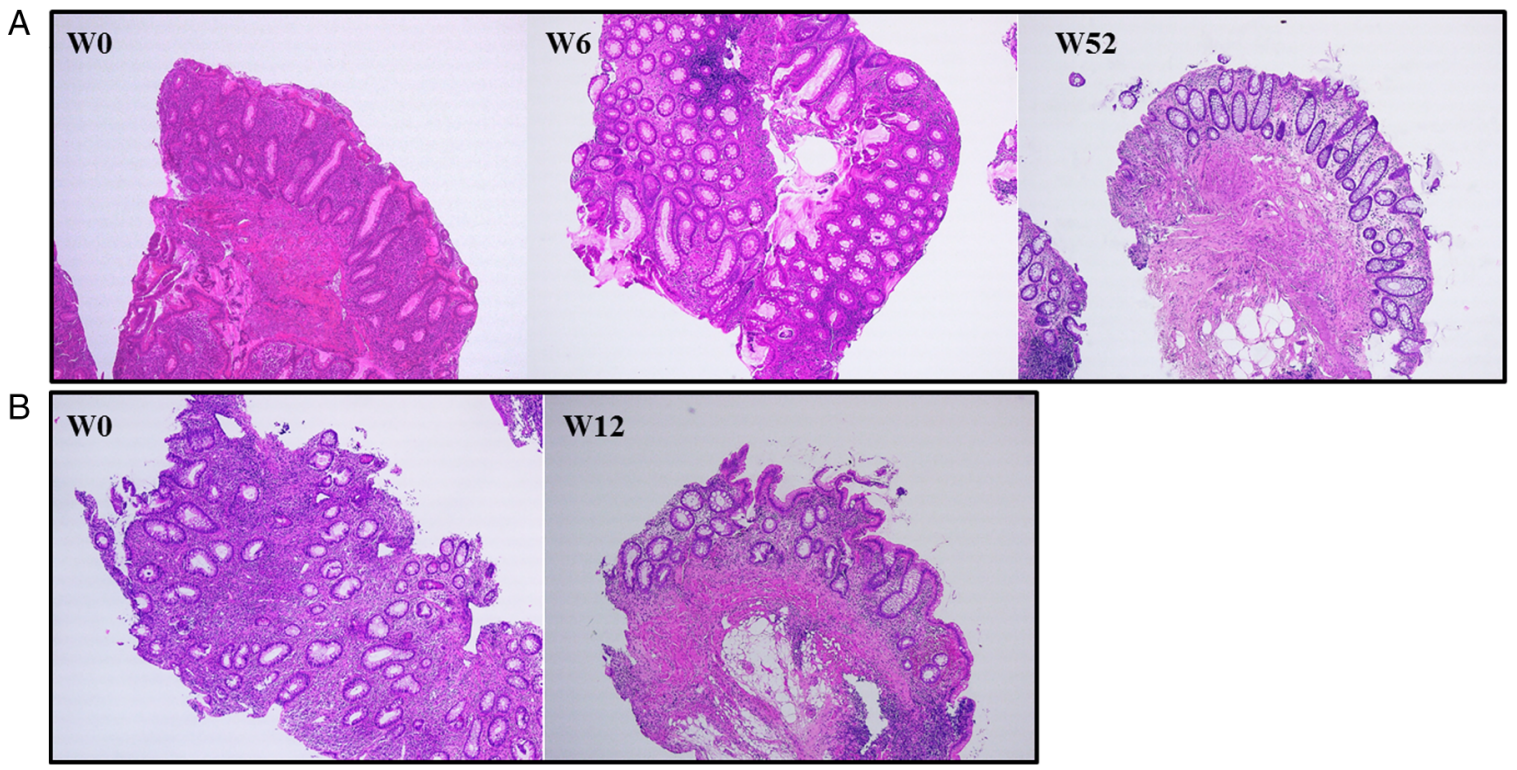

Figure 2 Histological changes in colonic biopsies of UC patients with mucosal healing after vedolizumab (VDZ) therapy (H\&E stain; original magnification $(0 \mathrm{M}) \times 50$ (detail OM $\times 100)$ ). (A) H\&E stainings from a UC patient with endoscopic and histological healing at week (W)6 and W52 after VDZ therapy. W0, moderately active erosive UC (Geboes score 5.3); W6, epithelial restoration, decrease of the mononuclear cell infiltrate, no residual neutrophils (Geboes score 1.1); W52, intact mucosa, hypocellular lamina propria (Geboes score 1.1). (B) H\&E stainings from a UC patient with endoscopic and histological healing at W12 after VDZ therapy. W0, dense mixed inflammatory infiltrate with crypt destruction (Geboes score 5.1); W12, patchy residual mononuclear cell infiltrate, no neutrophils (Geboes score 1.1).

UC who achieved endoscopic mucosal healing (= responders) with VDZ at W6/W12/W52 or with IFX at W4-6 clustered together with the control samples, whereas the pretreatment (W0) samples from active patients with UC and the samples from UC patients without endoscopic mucosal healing (= nonresponders) after VDZ or IFX therapy grouped together.

VDZ restored colonic expression of many immune-related genes in responders at W52

Colonic gene expression profiles before VDZ therapy were compared with the profiles after VDZ therapy and also with a cohort of patients treated with IFX (see online supplementary table S2).

In VDZ non-responders, no genes were differentially expressed at W6, W12 or W52 compared with their baseline samples. In parallel to this observation, we also observed no effect on colonic gene expression at W4-6 in IFX nonresponders compared with their baseline samples.
In VDZ responders (figure 4), no gene probe sets changed significantly by $\mathrm{W} 6$ and only five changed significantly by W12 (\: IDO1, REG3A, KLK6, SAA2 and $\nearrow: P C K 1)$ compared with W0. By W52, however, many significant gene expression differences were observed in VDZ responders compared with W0: 593 gene probe sets were either downregulated $(n=462)$ or upregulated $(\mathrm{n}=131)$, and $375(63 \%)$ of these gene probe sets overlapped with those identified in IFX responders at W4-6 vs W0 (figure 4). These represented genes are mainly involved in cellular movement, immune cell trafficking, haematological system development and function, and inflammatory response with cellular movement/migration/chemotaxis/homing of blood cells (leucocytes) as top significant biological subfunctions (see online supplementary table S3). VDZ had a unique effect at W52 in responders on the expression of LPHN2 (\), FGF7

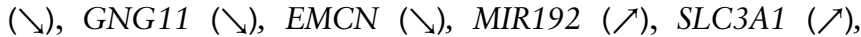
FABP6 $(\nearrow)$ and MEP1B $(\nearrow)$. In contrast, IFX had no effect on

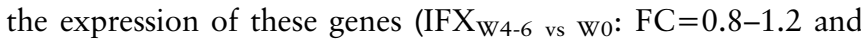
FDR >0.2). Interestingly, the baseline (= W0) expression levels

Table 2 Histological examination in biopsy samples of responders showing endoscopic and histological healing at week (W)6/W12/W52 by vedolizumab (VDZ) or at W4-6 by infliximab (IFX) and their baseline (W0) samples

\begin{tabular}{|c|c|c|c|c|c|c|}
\hline Histological parameters & VDZ W0 $(n=10)$ & VDZ W6 $(n=3)$ & $\begin{array}{l}\text { VDZ W12 } \\
(n=2)\end{array}$ & VDZ W52 (n=7) & IFX W0 (n=5) & IFX W4-6 $(n=5)$ \\
\hline $\begin{array}{l}\text { (1) Architectural changes } \\
\text { (Geboes grade 0) }\end{array}$ & $\begin{array}{l}0.1: n=3(30 \%) ; 0.2: \\
n=7(70 \%)\end{array}$ & $\begin{array}{l}0.1: n=2(66.7 \%) ; \\
0.2: n=1(33.3 \%)\end{array}$ & $\begin{array}{l}0.2: n=2 \\
(100 \%)\end{array}$ & $\begin{array}{l}0.0: n=1(14.3 \%) ; 0.1: n=5 \\
(71.4 \%) ; 0.2: n=1 \quad(14.3 \%)\end{array}$ & $\begin{array}{l}0.1: n=1(20 \%) ; 0.2: \\
n=2(40 \%) ; 0.3: n=2 \\
(40 \%)\end{array}$ & $\begin{array}{l}0.1: n=2(40 \%) ; \\
0.2: n=3(60 \%)\end{array}$ \\
\hline $\begin{array}{l}\text { (2) Mononuclear infiltrate } \\
\text { (Geboes grade 1) }\end{array}$ & $\begin{array}{l}\text { 1.1: } n=2(20 \%) ; 1.2: \\
n=4(40 \%) ; 1.3: n=4 \\
(40 \%)\end{array}$ & $\begin{array}{l}\text { 1.0: } n=1(33.3 \%)_{i} \\
1.1: n=2(66.7 \%)\end{array}$ & $\begin{array}{l}1.1: n=2 \\
(100 \%)\end{array}$ & $\begin{array}{l}\text { 1.0: } n=2(28.6 \%) ; 1.1: n=5 \\
(71.4 \%)\end{array}$ & $\begin{array}{l}\text { 1.2: } n=1(20 \%) ; 1.36: \\
n=4(80 \%)\end{array}$ & $\begin{array}{l}\text { 1.1: } n=3(60 \%) \\
1.2: n=2(40 \%)\end{array}$ \\
\hline (3) $\mathrm{CD}^{+} \mathrm{T}$ lymphocytes & フノノ & 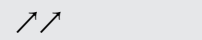 & $\nearrow \nearrow$ & $\nearrow$ & クノ & 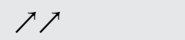 \\
\hline (4) $\mathrm{CD}^{+} / \mathrm{CD}^{+} \mathrm{T}$ cells & Normal & Normal & Normal & Normal & Normal & Normal \\
\hline $\begin{array}{l}\text { (5) Mast cells in lamina } \\
\text { propria }\end{array}$ & Normal & Normal & Normal & Normal & Normal & Normal \\
\hline
\end{tabular}


Figure 3 Principal component (PC) analysis of the filtered ( 5585 gene probe sets) $\log 2$ microarray data of control, UC week (W)0, UC vedolizumab (VDZ) W6/W12/W52 and UC infliximab (IFX) W4-6 colonic mucosal samples. A two-principal component plot is shown with the first component along the $x$-axis and the second component along the $y$-axis. The percentage of variability explained by each component (between the brackets) is shown on the axis. NR, non-responders, $\mathrm{R}$, responders.

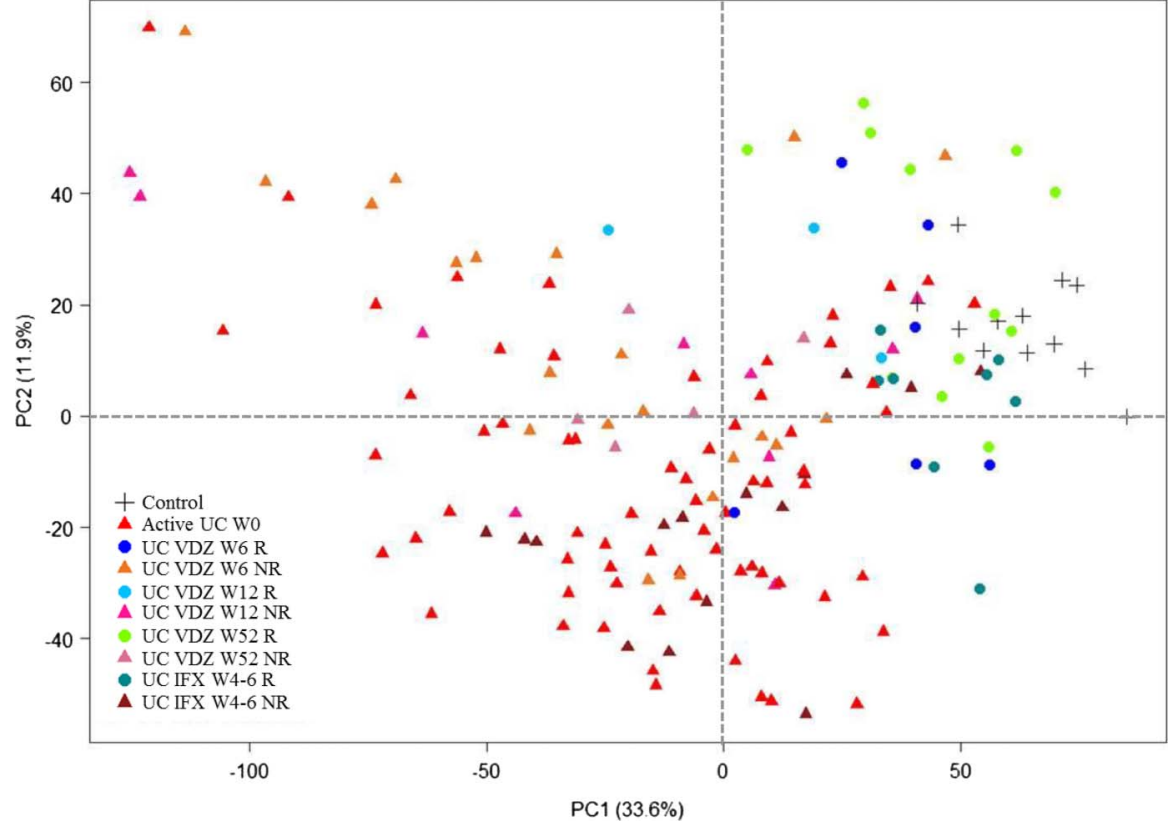

of LPHN2 and FGF7 were significantly lower in IFX responders compared with IFX non-responders (see online supplementary table S2).

In contrast to our previous gene expression study in patients treated with IFX, ${ }^{12}$ we could not identify genes predictive for response to VDZ by comparing the pre-VDZ treatment (W0) array profiles of responders with non-responders (see online supplementary table S2).

\section{Persistent dysregulation of colonic gene expression after VDZ and} IFX therapy in UC responders

Next, we compared the colonic gene expression microarray profiles of the responders after therapy with the profiles of the controls. Many gene probe sets remained significantly dysregulated in both VDZ and IFX responders compared with healthy controls (see online supplementary table S2). A large overlap of these persistently dysregulated genes was seen between VDZ and IFX (figure 5). The biofunctional analyses of the persistently dysregulated genes showed a common predominance of the biological (sub-)functions cellular movement/immune cell trafficking/haematological system development and function (cell movement/infiltration of leucocytes, eg, $\nearrow: C 2, I L 1 B$, PTGS2, TIMP1, CCL20, FOS), lipid metabolism/molecular transport/small molecule biochemistry (concentration of triacylglycerol/quantity of steroid, eg, $\nearrow: E G R 1, I L 1 B, N R 4 A 1$, PTGS2 and \: AMACR, AQP8, VLDLR), cell death and survival (necrosis, eg, \: EGR1, FOS, GDF15, IL1B, NR4A1, PTGS2, TIMP1 and \: ABCG2, AMACR), and cell cycle (cell cycle progression, eg, $\nearrow: A R E G, E G R 1, F O S$, NR4A1, SERPINB5, TIMP1 and \: BRINP3) (see online supplementary table S3).

\section{Validation of microarray data by qRT-PCR}

From the microarray data, LPHN2, FGF7, IDO1 and IL8 were selected to confirm their change in colonic mucosal gene expression in (un-)treated patients with UC versus controls by qRT-PCR (figure 6). Consistent with the microarray data, the expression levels of the four genes were all significantly increased in active colonic mucosa before VDZ/IFX therapy (W0) versus control colons. After VDZ therapy, the colonic expression of these genes remained significantly increased at W12 in VDZ responders compared with control colons, and their expression levels returned to control levels only at W52, except for LPHN2. A decrease in LPHN2 expression was observed in VDZ responsignificant $(p=0.108)$ by qRT-PCR. No significant effect of IFX therapy was seen at W4-6 on the expression level of LPHN2 and FGF7 expression in IFX responders, and the pre-IFX treatment expression levels of LPHN2 and FGF7 were significantly decreased in IFX responders versus IFX non-responders.

The gene probe set representing MADCAM1, the $\alpha 4 \beta 7$-integrin ligand, was excluded from the microarray analyses by the non-specific filtering because of variability across the microarray samples. qRT-PCR was however performed for were significantly increased before therapy in inflamed colon of UC patients with active disease versus controls. After VDZ therapy, MADCAM1 expression remained significantly increased at W12 in VDZ responders versus controls and returned to control levels at W52.

\section{Protein localisation of selected genes by immunohistochemistry}

Tissue sections obtained from patients before and after VDZ therapy were stained by immunohistochemistry to investigate the localisation and cellular expression of MADCAM1, ITGB7 and LPHN2 (figure 7).

MADCAM1 was expressed in the blood vessel (most likely postcapillary venular) endothelium, with a more extensive and stronger expression in untreated and inflamed UC colonic mucosa (especially in granulation tissue) (figure $7 \mathrm{~B}$ ), versus normal mucosa (figure 7A). In VDZ responders, MADCAM1 expression was low in mucosal biopsies at W52 (figure 7C).

ITGB7 showed a membrane-accentuated staining in LP mononuclear cells (mostly macrophages and plasma cells) in the colonic mucosa. A more extensive staining was seen in untreated and inflamed UC mucosa (figure 7E) compared with normal mucosa (figure 7D), and this was mainly due to an increased density of the inflammatory cell infiltrate. In VDZ responders, the density of LP mononuclear cells decreased at W52, and this resulted in a decreased ITGB7 expression (figure 7F). ders at W52 vs W0; however, this decrease was not found to be MADCAM1 (figure 6E). The expression levels of MADCAM1 


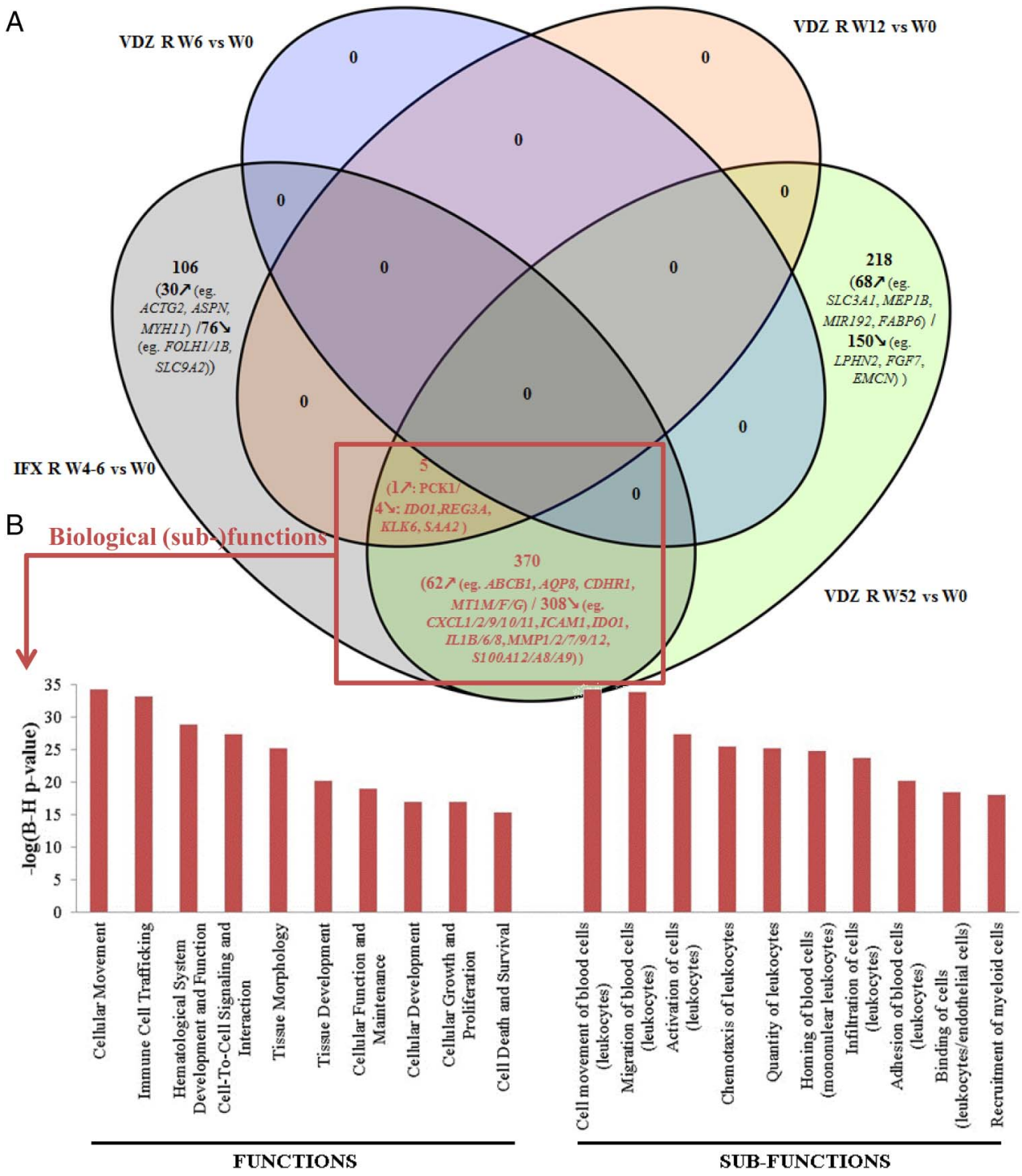

Figure 4 (A) Venn diagram of the significant gene probe sets in responders to vedolizumab (VDZ) or infliximab (IFX). (B) Bar chart represents the top 10 most significant biological (sub-)functions associated with the list of the 375 significant gene probe sets that overlapped between VDZ and IFX in the comparisons of responders before versus after therapy.

Inflamed and untreated UC mucosa showed membranous LPHN2 staining in smooth muscle cells of the muscularis mucosae and in the wall of small blood vessels (probably pericytes) (figure $7 \mathrm{H}$ ). Diminished vessel density or congestion in healed UC mucosa after VDZ therapy may partially explain decreased LPHN2 staining (figure 7I).

\section{DISCUSSION}

The efficacy of anti-tumour necrosis factor (TNF) therapy in IBD has pushed our therapy goals significantly, and endoscopic healing is now considered the required end point. Nevertheless, endoscopic healing is not curative and relapses are still observed in a significant proportion of patients, even when treatment is continued. This has led clinicians to challenge the concept of deep remission and explore whether histological or transmural healing (in the case of CD) could be a better end point and whether this can be achieved with our current drugs.

In this study, we investigated the role of histological healing following VDZ. VDZ is the first biological therapy with a new mode of action of selectively blocking leucocyte trafficking in the gut. We found that $55 \%$ of patients with UC who achieve endoscopic healing with VDZ at the studied time points also achieved histological healing. In contrast to patients who did not achieve mucosal healing, histological inflammatory features improved substantially in patients with mucosal healing after VDZ with a significant reduction in the inflammatory cell infiltrate. On the other hand, detailed histological examination also demonstrated that some degree of architectural abnormalities and of mononuclear inflammatory cell infiltrate persists. This indicates that the epithelial/mesenchymal architecture and the inflammatory cell composition need time to recover completely. The onset of the histological changes was observed as early as W6, although abnormalities were still present at W12 and the maximal effect was seen at W52. In the clinical setting, the onset of clinical response to VDZ may take longer than 6 weeks as shown in GEMINI I and in some patients even longer than 12 weeks.

Second, we investigated the effect of VDZ on the colonic mucosal gene expression in patients with UC using a wholegenome gene expression array. We compared our findings with those observed with IFX. The expression analysis first demonstrated that VDZ restored colonic expression of many genes 




mainly involved in leucocyte migration in patients with endoscopic healing at W52, but not yet at W6 and at W12. The majority of gene expression changes observed in VDZ healers at W52 was similar to those seen with IFX at W4-6, suggesting similar mechanisms of action for barrier and tissue restoration for both therapies although starting at different time points. Previously, it was shown that IFX, apart from its TNF- $\alpha$ neutralisation, has several other downstream effects in IBD. ${ }^{13}$ IFX not only induces apoptosis of activated intestinal mucosal $\mathrm{T}$ cells, but also restores the gut barrier. IFX deactivates the mucosal endothelium and thereby inhibits continued $\mathrm{T}$ cell recruitment. Moreover, IFX affects myofibroblast migration and thereby facilitates intestinal wound healing. The combination of all these events probably causes an early noticeable change at the mucosal gene expression level. VDZ, in contrast, only blocks T lymphocyte migration to the colonic mucosa and does not influence activated T lymphocytes already present in the LP, probably explaining its delayed effect. The latter was also confirmed by histological examination of the biopsies of patients with endoscopic and histological healing whereby an increased mononuclear cell infiltrate and a marked increase of $\mathrm{T}$ lymphocytes in the colonic mucosa were still visible at W6 and W12. Physicians have been discussing optimal bridging strategies for the delayed onset of action of VDZ and some have suggested that anti-TNF may fulfil this role. The gut selectivity of VDZ certainly is anticipated to carry advantages with respect to safety.

Although many gene expression changes overlapped between VDZ and IFX, the colonic expression of a subset of genes (eg, \: LPHN2, FGF7, EMCN, GNG11 and $\nearrow:$ MIR192, SLC3A1, FABP6, MEP1B) was uniquely influenced at W52 by VDZ, and not at W4-6 by IFX. We previously published that baseline expression of LPHN2 and FGF7 was decreased in IFX responders versus IFX non-responders, suggesting that these could be used as predictive markers for response to IFX therapy ${ }^{12}$ We feel these are important data and a possible next step to personalised therapy, as based on our findings, anti-TNF-naive patients with UC refractory to standard therapy who express high levels of LPHN2 or FGF7 would be better candidates for VDZ treatment than anti-TNF treatment. LPHN2 or latrophilin 2 is a member of the family of adhesion G-protein-coupled receptors that is characterised by a large N-terminus, which contains motifs identified in proteins involved in cell adhesion. ${ }^{14}$ Its function and tissue distribution are as yet insufficiently studied. Fibroblast growth factor 7 (FGF7) (also termed keratinocyte growth factor) is an epithelial cell-specific mitogen. Prior studies ${ }^{10}{ }^{15}$ have shown that the expression of FGF7 is increased in IBD colonic tissue and that FGF7 may play role in the mucosal repair in IBD. In contrast with the previously identified predictive gene signature for (non-)response to IFX in $\mathrm{UC},{ }^{12}$ we could not identify genes predictive for response to VDZ.

In non-responders to VDZ and IFX therapy, no effect of both therapies on the colonic mucosal gene expression was found. However, in CD, Leal et $a l^{16}$ showed by whole-genome transcriptional analysis in colonic mucosa that anti-TNF therapy downregulates a subset of inflammatory genes even in patients who do not achieve endoscopic remission.

As the triggers for recurrence of inflammation are unknown, we also investigated which biological pathways were involved in disease relapse by comparing the gene expression profiles of the VDZ and IFX responders with those of non-IBD controls. We found that the expression of many genes mainly involved in immune cell trafficking and lipid metabolism remained abnormal after therapy in patients who achieved endoscopic mucosal healing after VDZ or IFX therapy. These data are consistent with the histological findings whereby residual alterations were also seen. These persistent abnormalities might explain why mucosal lesions recur very early when patients do not receive maintenance therapy. Planell et $a l^{17}$ also described a permanently dysregulated state of mainly genes expressed by epithelial cells in the mucosa of patients with UC in remission. In accordance with the latter study, the epithelial cell expressed genes, 

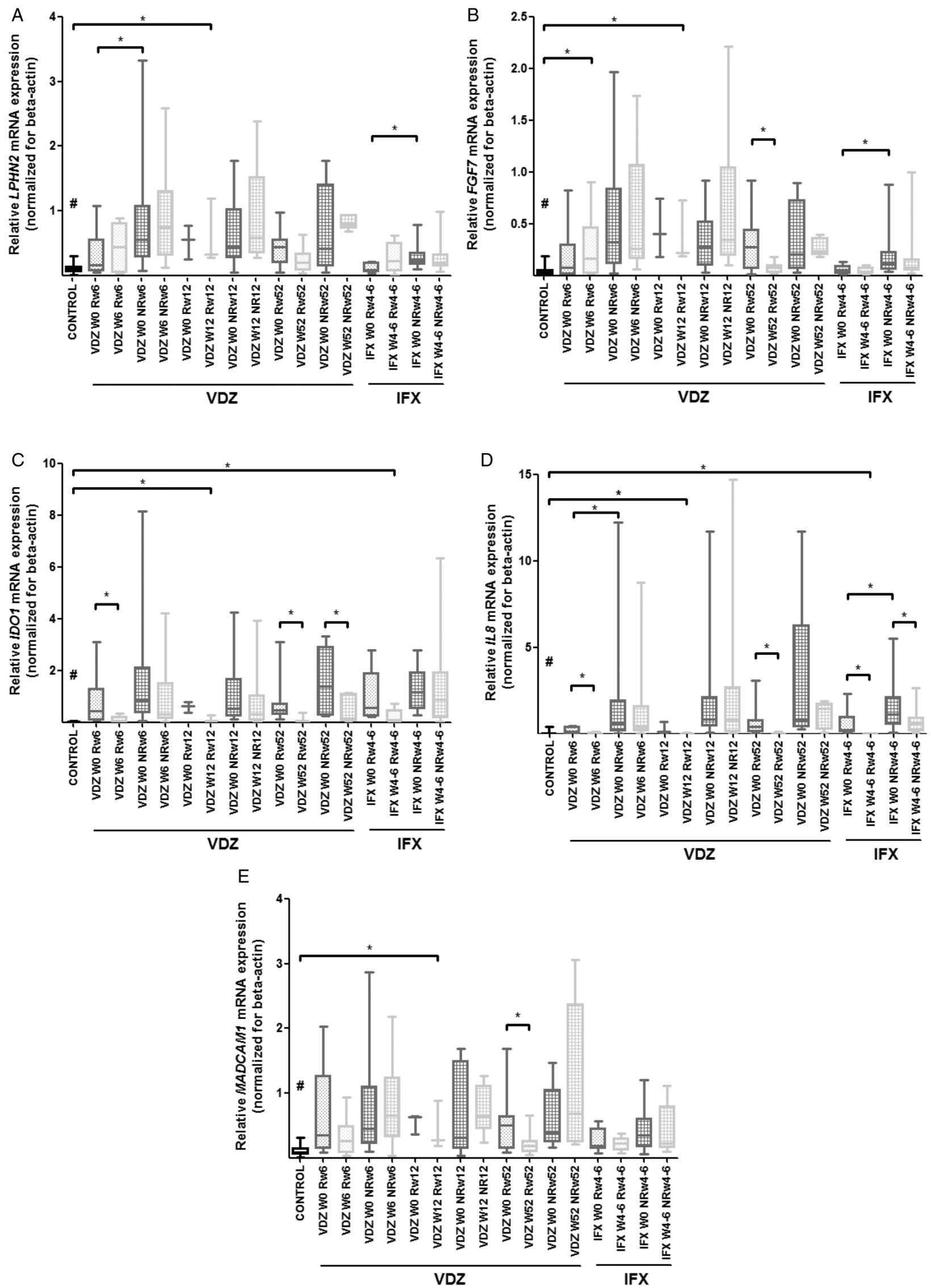

Figure 6 Box plots representing the qRT-PCR gene expression data of LPHN2 (A), FGF7 (B), IDO1 (C), IL8 (D) and MADCAM1 (E) in controls and patients with UC before and after therapy with vedolizumab (VDZ) or infliximab (IFX). Black, dark and light grey coloured box plots represent controls, responders (R) and non-responders (NR), respectively. Box plots with a dot or square pattern represent respectively before and after therapy. * significant; \#significant in UC week (W)0 vs controls. 


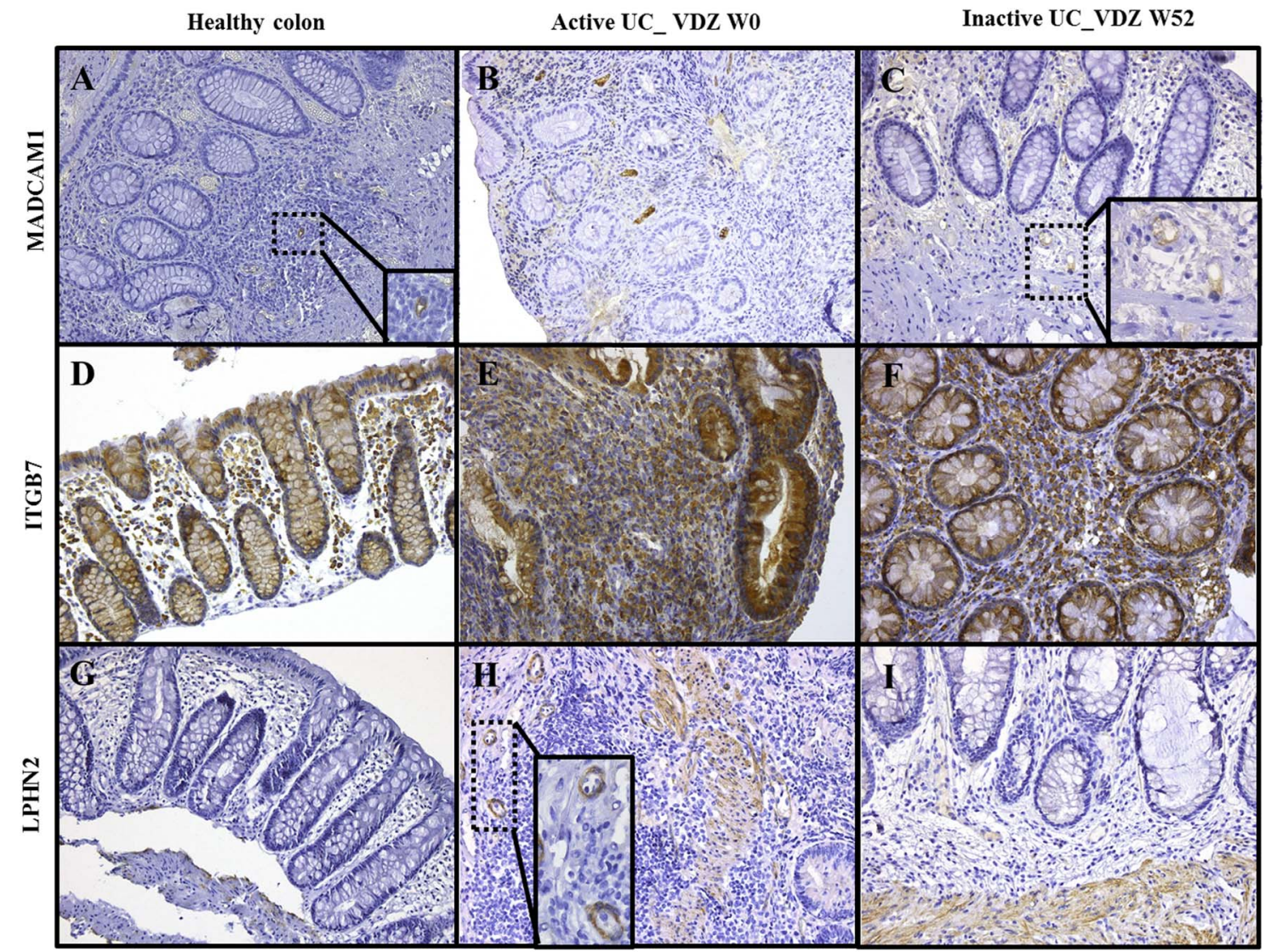

Figure 7 Immunohistochemistry for detection and localisation of MADCAM1 (A-C), ITGB7 (D-F) and LPHN2 (G-I) in paraffin-embedded, formalin-fixed tissue sections from colonic biopsies from control individuals $(A, D, G)$, patients with active UC before vedolizumab (VDZ) therapy $(B, E, H)$ and inactive patients with UC at week (W)52 after VDZ therapy $(C, F, I)$ (original magnification $(0 M) \times 200($ detail $0 M \times 400)$ ).

ABCG2, REG4, TFF1, SERPINB5, AQP8, RUNDC3B and $C H I 3 L 1$, also remained altered after therapy in the colonic mucosa of both VDZ and IFX responders. Furthermore, the significant overlap of persistently dysregulated genes in VDZ and IFX responders suggests that yet unidentified triggers of inflammation are incompletely blocked by these biological agents. These triggers can be environmental factors including damaging dietary components or microbial factors.

Our study has limitations: the patients treated with VDZ were all previously treated with IFX, and maybe even better results could be obtained in anti-TNF-naive patients. Second, we could not compare our results with a late time point (W52) for IFX. However, a similar effect observed by VDZ at W52 was seen by IFX at W4-6. Despite these limitations, we present the first data on histological improvement following VDZ therapy and important results were generated that may help in understanding the relative slow onset of clinical efficacy. The expression data of LPHN2 and FGF7 are interesting in view of the search for predictive markers of response to one or the other class but needs confirmation.

In conclusion, histological healing is achieved in patients with UC treated with VDZ, with a maximal effect seen at W52. Furthermore, VDZ restores, although incompletely, the colonic expression of many immune-related genes in patients with UC who achieve endoscopic healing at W52. However, persistent histological and gene expression abnormalities remain after therapy in patients who achieve mucosal healing. These observations may be related to disease recurrence when patients discontinue the treatment, thereby indicating that maintenance therapy with VDZ is necessary to control the ongoing intestinal inflammation.

\section{Author affiliations}

${ }^{1}$ Department of Clinical and Experimental Medicine, Translational Research Center for Gastrointestinal Disorders, KU Leuven, Leuven, Belgium

${ }^{2}$ Faculty of Medicine and Life Sciences, Hasselt University, Hasselt, Belgium ${ }^{3}$ Jessa Hospital, Hasselt, Belgium

${ }^{4}$ Department of Imaging \& Pathology, Translational Cell \& Tissue Research, KU Leuven, Leuven, Belgium

${ }^{5}$ Gene Expression Unit, Department of Cellular and Molecular Medicine, KU Leuven, Leuven, Belgium

${ }^{6}$ Department of Microbiology and Immunology, Rega Institute for Medical Research, KU Leuven, Leuven, Belgium

${ }^{7}$ Department of Human Genetics, University Hospitals Leuven, KU Leuven, Leuven, Belgium

${ }^{8}$ Department of Gastroenterology and Hepatology, University Hospitals Leuven, KU Leuven, Leuven, Belgium

Acknowledgements The authors thank Vera Ballet and Jan Van der Goten for their support in the collection of patient data and samples.

Contributors IA and GDH contributed equally to the article. IA: study concept and design, acquisition of data, analysis and interpretation of data, drafting of the manuscript, statistical analysis and technical support. GDH: study concept and design, analysis and interpretation of data and drafting of the manuscript. BL, LVL and WV: technical support. MdB and KM: critical revision of the manuscript for important intellectual content and technical support. IC, MF and GVA: critical revision of the manuscript for important intellectual content. FS: material support and critical revision of the manuscript for important intellectual content. PR: study concept and design, acquisition of data, analysis and interpretation of data, material support, critical revision of the manuscript for important intellectual content, obtained funding and study supervision. SV: study concept and design, acquisition of data, analysis and interpretation of data, material support, drafting of the manuscript, obtained funding and study supervision. 
Funding This work was supported by grants from the Fund for Scientific Research-Flanders (FWO-Vlaanderen; no. G.0440.06 and no. G.0479.10), and from the Belgian Inflammatory bowel disease Research and Development (BIRD). MF, GVA and SV are senior clinical researchers of FWO. MdB is supported by a grant of the Agency for Innovation by Science and Technology in Flanders.

Competing interests GDH—consulting fees: Genentech, Centocor, Galapagos. MF-grant support: Takeda; lecture fees: Abbvie, Boehringer-Ingelheim, Chiesi, Falk, Ferring, Janssen, Mitsubishi Tanabe, MSD, Takeda, Tillotts, Zeria; consulting fees: Abbvie, Boehringer-Ingelheim, Ferring, Janssen, MSD. GVA — grant support: Abbvie, MSD; lecture fees: Abbvie, Ferring, MSD, Janssen, Takeda; consulting fees: Abbvie, MSD, Takeda. PR — grant support: Abbvie, Centocor, Merck, UCB; lecture fees: Centocor, Merck, Abbvie; consulting fees: Centocor, Merck, UCB, Abbvie, Millenium/ Takeda, Genentech/Hoffman LaRoche, Merck/Serono, Bristol Myers Squibb, Robarts, Tillotts, Pfizer, Falk Pharma. SV—grant support from Abbvie, MSD; lecture fees: Abbvie, MSD, Takeda, Ferring, Falk Pharma, Hospira, Tillotts; grant support from Abbvie, MSD; consulting fees: Abbvie, MSD, Takeda, Ferring, Genentech/Roche, Shire, Pfizer, Galapagos, Mundipharma, Hospira, Celgene, Second Genome, Janssen.

Patient consent Obtained.

Ethics approval The ethics committee of the Leuven University Hospitals approved the study (B322201213950/S53684).

Provenance and peer review Not commissioned; externally peer reviewed.

\section{REFERENCES}

1 Feagan BG, Rutgeerts $P$, Sands BE, et al. Vedolizumab as induction and maintenance therapy for ulcerative colitis. N Engl J Med 2013;369:699-710,

2 Sandborn WJ, Feagan BG, Rutgeerts $P$, et al. Vedolizumab as induction and maintenance therapy for Crohn's disease. N Engl J Med 2013;369:711-21.

3 Neurath MF. New targets for mucosal healing and therapy in inflammatory bowel diseases. Mucosal Immunol 2014;7:6-19.

4 Bessissow $T$, Lemmens $B$, Ferrante $M$, et al. Prognostic value of serologic and histologic markers on clinical relapse in ulcerative colitis patients with mucosal healing. Am J Gastroenterol 2012;107:1684-92.
5 Geboes K, Riddell R, Ost A, et al. A reproducible grading scale for histological assessment of inflammation in ulcerative colitis. Gut 2000;47:404-9.

6 Rutgeerts P, Sandborn WJ, Feagan BG, et al. Infliximab for induction and maintenance therapy for ulcerative colitis. N Engl J Med 2005;353:2462-76.

7 Breynaert C, Dresselaers T, Perrier C, et al. Unique gene expression and MR T2 relaxometry patterns define chronic murine dextran sodium sulphate colitis as a model for connective tissue changes in human Crohn's disease. PLOS ONE 2013;8: e68876.

8 Bengtsson R, Simpson K, Bullard J, et al. aroma.affymetrix: a generic framework in $R$ for analyzing small to very large Affymetrix data sets in bounded memory. Tech Report \#745. Berkeley: Department of Statistics, University of California, 2008.

9 Smyth GK. Linear models and empirical bayes methods for assessing differential expression in microarray experiments. Stat App/ Genet Mol Biol 2004;3:Article3.

10 Arijs I, De Hertogh G, Machiels K, et al. Mucosal gene expression of cell adhesion molecules, chemokines, and chemokine receptors in patients with inflammatory bowel disease before and after infliximab treatment. Am I Gastroenterol 2011;106:748-61.

11 Pfaffl MW. A new mathematical model for relative quantification in real-time RT-PCR. Nucleic Acids Res 2001;29:e45.

12 Arijs I, Li K, Toedter G, et al. Mucosal gene signatures to predict response to infliximab in patients with ulcerative colitis. Gut 2009;58:1612-19.

13 Danese S. Mechanisms of action of infliximab in inflammatory bowel disease: an anti-inflammatory multitasker. Dig Liver Dis 2008;40(Suppl 2):S225-8.

14 Boucard AA, Maxeiner S, Südhof TC. Latrophilins function as heterophilic cell-adhesion molecules by binding to teneurins: regulation by alternative splicing. J Biol Chem 2014;289:387-402.

15 Finch PW, Cheng AL. Analysis of the cellular basis of keratinocyte growth factor overexpression in inflammatory bowel disease. Gut 1999;45:848-55

16 Leal RF, Planell N, Kajekar R, et al. Identification of inflammatory mediators in patients with Crohn's disease unresponsive to anti-TNFalpha therapy. Gut 2015;64:233-42.

17 Planell N, Lozano JJ, Mora-Buch R, et al. Transcriptional analysis of the intestinal mucosa of patients with ulcerative colitis in remission reveals lasting epithelial cell alterations. Gut 2013;62:967-76. 